\title{
Correlation between efficacy of PSK postoperative adjuvant immunochemotherapy for gastric cancer and expression of MHC class I
}

\author{
GENTARO ITO, HIROAKI TANAKA, MASAICHI OHIRA, MAMI YOSHII, KAZUYA MUGURUMA, \\ NAOSHI KUBO, MASAKAZU YASHIRO, NOBUYA YAMADA, KIYOSHI MAEDA, \\ TETSUJI SAWADA and KOSEI HIRAKAWA
}

\begin{abstract}
Department of Surgical Oncology, Osaka City University Graduate School of Medicine, Abeno-Ku, Osaka 545-8585, Japan
\end{abstract}
Received January 6, 2012; Accepted February 13, 2012

DOI: $10.3892 /$ etm.2012.537

\begin{abstract}
Protein-bound polysaccharide K (PSK) is a glycoprotein that is purified from the mushroom Coriolus versicolor. In Japan, PSK is clinically used in combination with anticancer agents following gastric cancer surgery. Evaluation of the response is difficult, as efficacy is determined via antitumor immunoenhancing effects, and for that reason PSK has not become a standard therapy. The present study evaluated the expression of MHC class I in gastric cancer patients who received PSK postoperative adjuvant immunochemotherapy, and investigated the correlation between MHC class I expression and clinical outcomes. The subjects comprised 349 patients with stage II/III gastric cancer, who had received adjuvant therapy following curative resection between 1995 and 2008. MHC class I expression in the primary lesion was evaluated by immunohistochemical staining. Patients were divided into two treatment groups: one was only administered oral chemotherapy (chemotherapy-only group) and the other was administered chemotherapy plus PSK (PSK group). The clinical outcomes were compared between the two groups. The two groups did not differ in regard to their MHC class I expression. Expression-negative cases demonstrated 3-year recurrence-free survival (RFS) rates of $65 \%$ in the PSK group and $47 \%$ in the chemotherapy-only group. Therefore, the PSK group revealed a prolonged survival. For the 82 expressionnegative cases with $\mathrm{pN} 2$ or greater, the RFS rates were $68 \%$ in the PSK group and $28 \%$ in the chemotherapy-only group, representing a significant difference. Thus, PSK adjuvant immunochemotherapy may be effective in MHC class I-negative patients, who are in a state of antitumor immu-
\end{abstract}

Correspondence to: Dr Hiroaki Tanaka, Department of Surgical Oncology, Osaka City University Graduate School of Medicine, 1-4-3 Asahimachi, Abeno-Ku, Osaka 545-8585, Japan

E-mail: hiroakitan@med.osaka-cu.ac.jp

Key words: gastric cancer, MHC class I, PSK immunochemotherapy nological tolerance, and patients with advanced lymph node metastasis of $\mathrm{pN} 2$ or greater.

\section{Introduction}

In Japan, adjuvant therapy using S-1 is considered effective for stage II/III gastric cancer. However, postoperative recurrence is observed in $30 \%$ of stage III patients, and the efficacy of S-1 monotherapy is marginal (1). Thus, combination of S-1 with various anticancer agents is being tested. Protein-bound polysaccharide K (PSK) is a glycoprotein extracted from the mushroom Coriolus versicolor belonging to the Polyporaceae family (2). Use of this antitumor agent is approved by the Japanese health insurance system, and has been confirmed to prolong survival in gastric cancer patients. PSK is considered to demonstrate not only direct cytocidal activity, but also antitumor immunoenhancing effects $(3,4)$. However, the mechanisms of these effects remain unclear, and in addition, since 1994 no studies have reported any clinical efficacy of PSK against gastric cancer, thus this agent has not been recognized as a standard therapy (5).

The fact that hosts become immunocompromised at the time of tumor progression has long been considered to be significant. In particular, decreased expression of MHC class I by the tumor is one mechanism which allows evasion of cytotoxic T lymphocytes (CTLs), which play a central role in tumor immunity (6). This has been reported to correlate with a poor prognosis for various types of cancer, including gastric cancer (7-9).

We conducted a retrospective study of the efficacy of PSK adjuvant immunochemotherapy, in which we evaluated the expression of MHC class I in the primary lesions of patients with stage II/III gastric cancer, for which adjuvant chemotherapy is indicated.

\section{Patients and methods}

Patients. The subjects comprised 349 patients with stage II/III gastric cancer who had undergone adjuvant therapy following curative resection at Osaka City University, Osaka, Japan from 1995 to 2008. The indication for adjuvant therapy was stage II/ III disease; histological depth of invasion T2-T3, as stipulated 
in the Japanese Gastric Cancer Association guidelines (10). For 225 of the 349 patients, adjuvant chemotherapy consisting of only an oral fluoropyrimidine formulation was administered (chemotherapy-only group), while the remaining 124 patients were administered an oral fluoropyrimidine formulation as adjuvant chemotherapy, plus PSK as immunotherapy (PSK group). The adjuvant antitumor chemotherapy agent consisted of one of the following: S-1 (80-120 mg/body/day), UFT (300-600 mg/body/day), furtulon (800-1200 mg/body/ day) or 5-FU (200-300 mg/body/day). PSK was administered at $3 \mathrm{~g} / \mathrm{day}$ in combination with the antitumor agent. The overall median duration of chemotherapy administration was 19 months (range, 1-70 months). The median duration for each of the agents was 18 months (range, 5-48 months) for S-1, 19 months (range, 1-70 months) for UFT, 18 months (range, 2-60 months) for furtulon and 22 months (range, 6-36 months) for 5-FU. Follow-up examinations aimed at detecting recurrence consisted of gastroscopy, computed tomography (CT) and blood testing. These examinations were performed monthly for the first 3 months after discharge, and then at 6-month intervals. Informed consent was obtained from all patients in this study. The study protocol conformed to the ethical guidelines of the Declaration of Helsinki. This study was approved by the Osaka City University Ethics Committee.

Immunohistochemical staining. Immunohistochemical staining was performed on $4-\mu$ m paraffin-embedded sections of the primary gastric cancer lesion. The avidin-biotinyl-enzyme complex (ABC) method was used for immunostaining of MHC class I (7). The sections were first reacted with rabbit serum blocking reagent, and then with a 1:100 dilution of mouse anti-human HLA class I monoclonal antibody (Abcam, Cambridge, MA, USA) at $4^{\circ} \mathrm{C}$ for $24 \mathrm{~h}$. After reacting with a secondary antibody in a moist chamber, sections were stained with diaminobenzidine, and then nuclear-stained with hematoxylin.

Patients with tumor cell cytoplasm demonstrating $\geq 50 \%$ staining for MHC class I were considered to be positive for MHC class I expression, while all other patients were considered to be negative (Fig. 1).

Statistical analysis. All statistical analyses were performed using SPSS II software (SPSS, Chicago, IL, USA). Clinical pathological data were subjected to binomial logistics testing. Recurrence-free survival (RFS) periods were calculated using the Kaplan-Meier method, and the differences between the two groups were analyzed by the log-rank test. Multivariate analysis of outcomes was performed by Cox regression analysis. $\mathrm{P}<0.05$ was considered to indicate a statistically significant difference.

\section{Results}

Patient background and clinical outcomes. The mean duration of follow-up for the total 349 patients was 49 months (range, 2-148 months), and the 5-year RFS was 64\%. Recurrence was diagnosed in 147 patients, occurring after a median of 27 months. The initial recurrence consisted of peritoneal dissemination in 44 patients, liver metastasis in 42 and lymph node recurrence in 32 . The type of oral anti-
Table I. Background of the 349 patients with stage II/III gastric cancer.

\begin{tabular}{|c|c|c|c|}
\hline $\begin{array}{l}\text { Clinicopathological } \\
\text { factors }\end{array}$ & $\begin{array}{l}\text { PSK group } \\
(\mathrm{n}=124)\end{array}$ & $\begin{array}{l}\text { Chemotherapy- } \\
\text { only group } \\
(\mathrm{n}=225)\end{array}$ & P-value \\
\hline $\begin{array}{l}\text { Age (years) } \\
(\text { mean } \pm \mathrm{SD})\end{array}$ & $62 \pm 10$ & $63 \pm 10$ & 0.128 \\
\hline Gender & & & 0.279 \\
\hline Male & 91 & 140 & \\
\hline Female & 33 & 85 & \\
\hline Histology & & & 0.415 \\
\hline Intestinal type & 49 & 93 & \\
\hline Diffuse type & 75 & 132 & \\
\hline pT & & & 0.264 \\
\hline pT1-T2 & 20 & 49 & \\
\hline pT3-T4 & 104 & 176 & \\
\hline $\mathrm{pN}$ & & & 0.311 \\
\hline Negative & 20 & 47 & \\
\hline Positive & 104 & 178 & \\
\hline Stage & & & 0.180 \\
\hline II & 46 & 98 & \\
\hline III & 78 & 127 & \\
\hline Ly & & & 0.591 \\
\hline Negative & 19 & 41 & \\
\hline Positive & 105 & 184 & \\
\hline $\mathrm{V}$ & & & 0.479 \\
\hline Negative & 79 & 154 & \\
\hline Positive & 45 & 71 & \\
\hline
\end{tabular}

PSK, protein-bound polysaccharide $\mathrm{K}$; SD, standard deviation; pT, depth of tumor invasion; T1, invasion until submucosa; $\mathrm{T} 2$, invasion until muscularis propria; $\mathrm{T} 3$, invasion until serosa; $\mathrm{T} 4$, invasion until adjacent structures; $\mathrm{pN}$, lymph node metastasis; Ly, lymphatic invasion; $\mathrm{V}$, venous invasion.

cancer agent administered had no effect on the outcome. No significant differences were found for background factors (age, gender, and tumor stage) of the 124 patients in the PSK group compared with the 225 patients in the chemotherapyonly (control) group (Table I). The 4 anticancer agents were administered to nearly the same number of patients. In addition, no difference was evident in the recurrence rates between the treatment groups. However, cases of hepatic recurrence outnumbered cases of peritoneal recurrence in the PSK group. The three-year RFS was $60 \%$ for the PSK group and $62 \%$ for the chemotherapy-only group, demonstrating no significant difference.

Correlation between expression of MHC class I and efficacy of PSK postoperative adjuvant immunochemotherapy. We next investigated the expression of MHC class I in the primary gastric cancer lesions and its correlation with the efficacy of PSK therapy. Immunohistochemical staining revealed MHC class I expression in the cytoplasm and cell membrane in the 
A

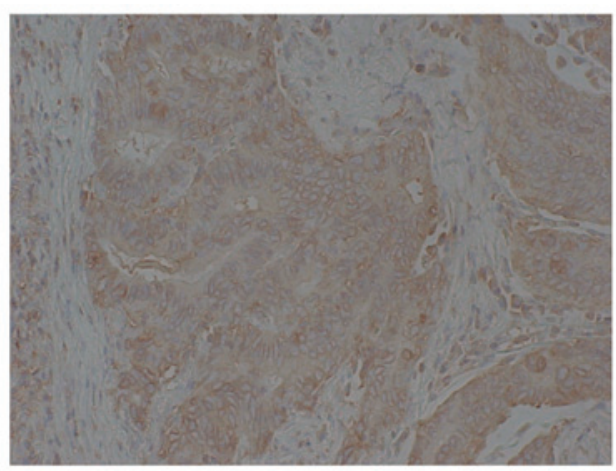

B

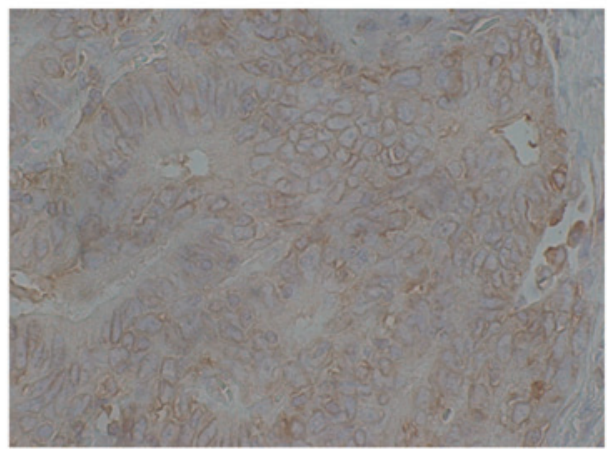

C

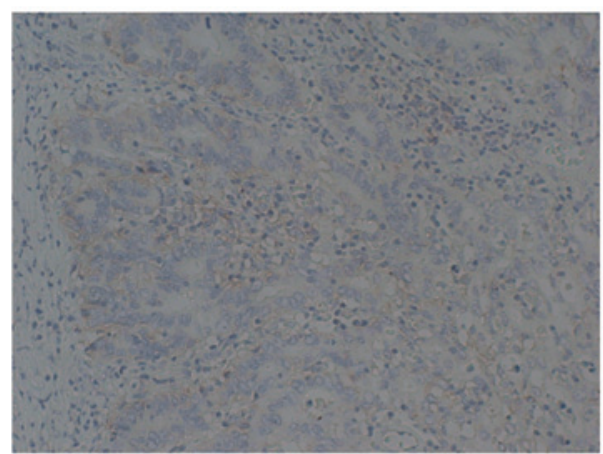

D

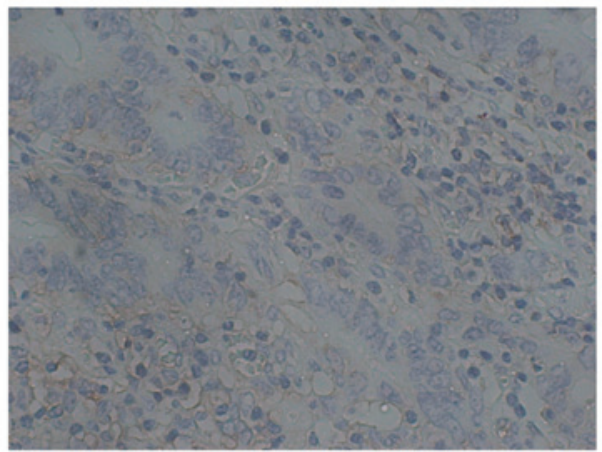

Figure 1. Immunohistochemical (IHC) staining of MHC class I in gastric cancer tissue. Expression of MHC class I in paraffin-embedded sections of human gastric cancer specimen as determined by IHC analysis. (A and B) IHC positive staining with anti-MHC class I antibody; (C and D) IHC negative staining with anti-MHC class I antibody. (A and C: magnfication, x200; B and D: magnification, x400).

Table II. Relative risk of recurrence in multivariate analysis.

\begin{tabular}{lccc}
\hline Variable category & Hazard ratio & $95 \%$ CI & P-value \\
\hline $\begin{array}{l}\text { Histology } \\
\text { (Intestinal/diffuse type) }\end{array}$ & 0.634 & $0.424-0.950$ & 0.027 \\
$\begin{array}{l}\text { Depth of tumor invasion } \\
\text { (pT1,2/3,4) }\end{array}$ & 2.935 & $1.566-5.55$ & 0.001 \\
$\begin{array}{l}\text { LN metastasis } \\
\text { (pN0/1,2,3) }\end{array}$ & 2.530 & $1.498-4.274$ & 0.001 \\
$\begin{array}{c}\text { MHC class I expression } \\
\text { (Positive/negative) }\end{array}$ & 0.444 & $0.304-0.674$ & $<0.001$ \\
\hline
\end{tabular}

pT, depth of tumor invasion; pN, number of lymph node metastases.

gastric cancer tissues. Immunohistochemical staining of $\geq 50 \%$ of tumor tissues was observed in 193 patients (55\%), who were considered to be positive for MHC class I expression. The remaining 156 patients (45\%) were considered to be negative for MHC class I expression (Fig. 1). MHC class I expression was significantly reduced in patients with diffuse-type histology.

In the patient cohort, the degree of differentiation of the primary lesion, depth of tumor invasion (pT), the number of lymph node metastases $(\mathrm{pN})$ and expression of MHC class I were identified as prognostic factors for recurrence (Table II). Prognostic outcomes were significantly worse for MHC class I expression-negative patients than for positive patients (Fig. 2A). This prognostic difference was prominent among the chemotherapy-only group (Fig. 2B). On the other hand, there was no significant difference in the PSK group (Fig. 2C). In the PSK group, venous invasion and differentiation of the primary tumor, but not expression of MHC class I, were prognostic factors.

MHC class I-positive patients in the the PSK group demonstrated relatively poorer prognosis than the chemotherapy-only group, but in the expression-negative patients the 3-year RFS was $62 \%$ in the PSK group, higher than the $50 \%$ in the chemotherapy-only group (Fig. 3). There were several differences in the patient backgrounds between the chemotherapy-only group and the PSK group in MHC class I-positive patients. The PSK group in the expressionpositive cohort revealed increased venous invasion compared to the chemotherapy-only group.

Thus, we hypothesized that PSK improved expression of MHC class I and induced antitumor immnological effects against remnant cancer cells. The prognostic factors (histological types, $\mathrm{pT}$ and $\mathrm{pN}$ ) and the related factor (venous invasion) were then compared with the clinical outcomes for the two treatment groups.

For three factors of histological types, venous invasion and pT, no statistically significance was observed between the PSK group and the chemotherapy-only group. However, the 3-year RFS was higher in the PSK group than that in the chemotherapy-only group (Fig. 4A-C). Significantly, for the $\mathrm{pN}$ factor, investigation by stratification of the number of lymph node metastases revealed no difference in the RFS between the treatment groups for $n 0$ and $n 1$ patients, whereas the RFS was significantly longer in the PSK group for patients with $\geq 3$ lymph node metastases (i.e., n2 or higher). The 3 -year RFS was $65 \%$ in the PSK group and $34 \%$ in the chemotherapy-only group (Fig. 4D). 

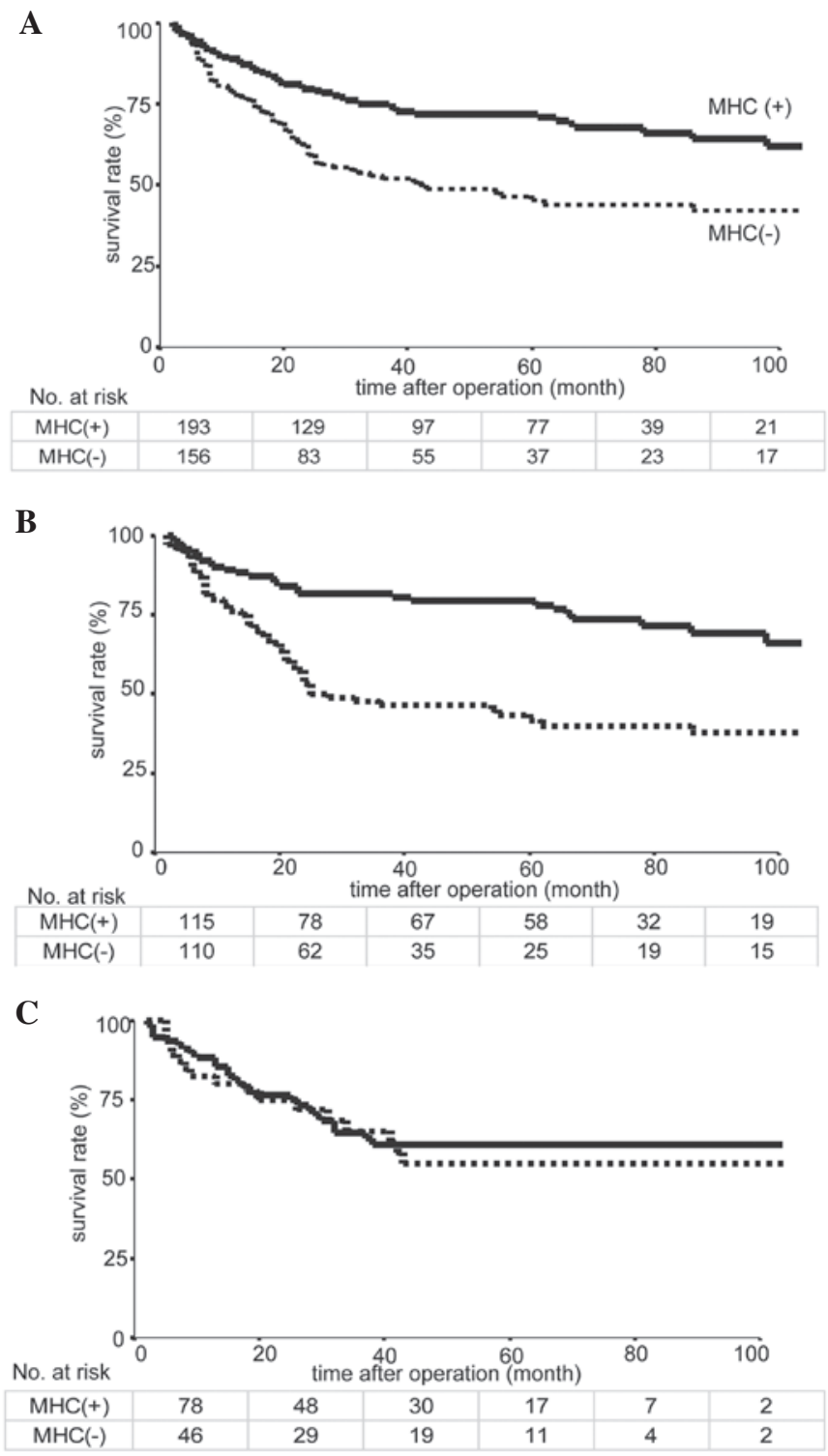

Figure 2. Comparison of relapse-free survival according to the expression of MHC class I. Patients were divided into MHC class I-positive cases ( $\mathrm{n}=193$, solid line) and -negative cases ( $\mathrm{n}=156$, dotted line). Survival curves (A) for all patients included in this study, (B) for the chemotherapy-only group and (C) for the PSK group. Relapse-free survival increased significantly in the MHC class I-positive patients compared to the negative patients in the total patient group and the chemotherapy-only group, but not in the PSK group. PSK, protein-bound polysaccharide K.

\section{Discussion}

The present results demonstrated that addition of PSK adjuvant immunochemotherapy to anticancer chemotherapy for patients who had undergone curative resection for gastric cancer improved the prognosis in patients with a gastric cancer primary lesion negative for MHC class I expression, and in patients with advanced lymph node metastasis. Therefore, PSK adjuvant immunochemotherapy has the potential to be effective in certain subgroups of gastric cancer patients.

Various studies have reported $\mathrm{T}$ factor, tumor diameter and tumor histology as prognostic factors for advanced gastric cancer (11). However, the most significant prognostic factor for patients who have undergone curative gastric resection
$\mathbf{A}$

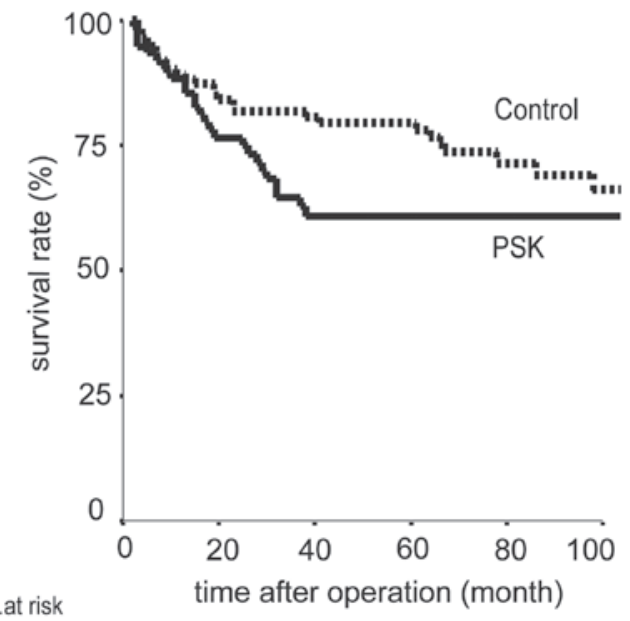

\begin{tabular}{|c|c|c|c|c|c|c|}
\hline No.at risk & \multicolumn{5}{c|}{ time after operation (month) } \\
\hline PSK & 78 & 48 & 30 & 18 & 7 & 2 \\
\hline Control & 115 & 78 & 67 & 58 & 32 & 19 \\
\hline
\end{tabular}

B

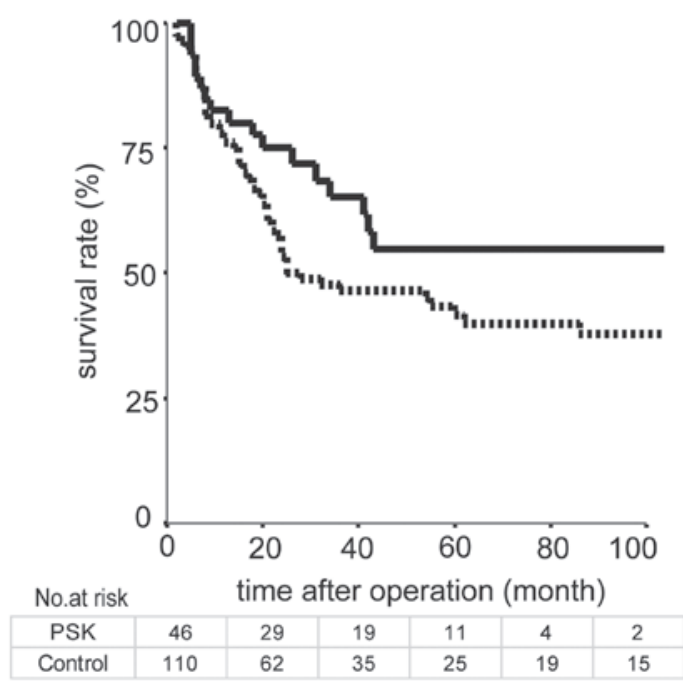

Figure 3. Comparison of relapse-free survival according to postoperative adjuvant therapy. Patients were divided into the PSK group $(n=124$, solid line) and the chemotherapy-only control group $(n=225$, dash line). Relapse-free survival in (A) MHC class I-positive and (B) -negative cases. Although the observed difference was not significant, these data suggest that PSK treatment may improve the prognosis of MHC class I-negative patients. PSK, protein-bound polysaccharide $\mathrm{K}$.

has been reported to be the number of metastatic lymph nodes (12-14). Prognosis is poor for patients with $\geq 3$ metastatic lymph nodes ( $\geq n 2)$, with a reported survival rate of 50\% (15). In accordance with the present cohort, the number of metastatic lymph nodes correlated with lymphatic invasion, depth of tumor invasion and diffuse-type histology (data not shown). Moreover, we identified that the number of metastatic lymph nodes represented an independent prognostic factor.

In the body, the mechanism of immunosurveillance plays a significant role in antitumor effects (16). Cancer progression and immunoevasion are known to be markedly correlated. CTLs play a central role in immune responses to cancer. Receptors on CTLs reportedly recognize MHC class I antigen complexes on the surface of cancer cells and attack them. However, expression of MHC class I on cancer cells is decreased. Decreased expression of MHC class I and a poor 
A

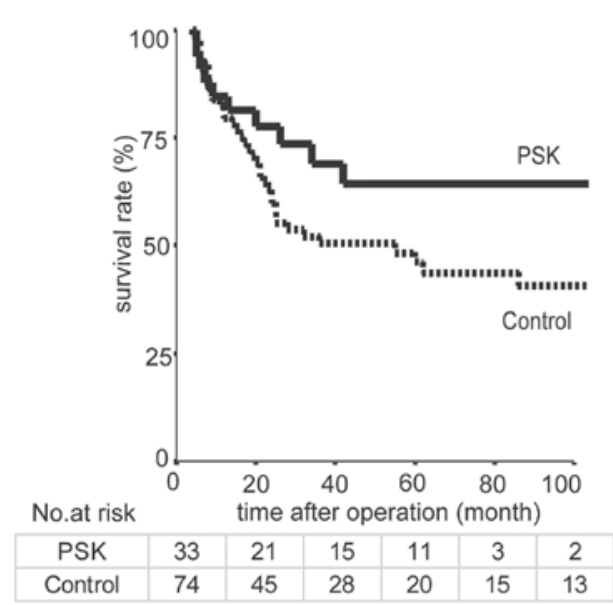

B

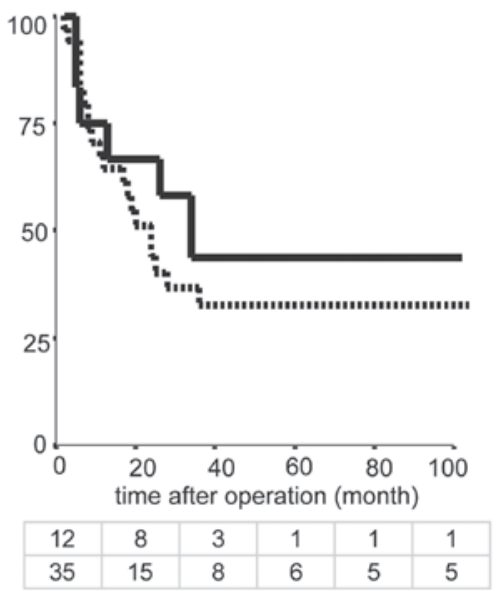

D

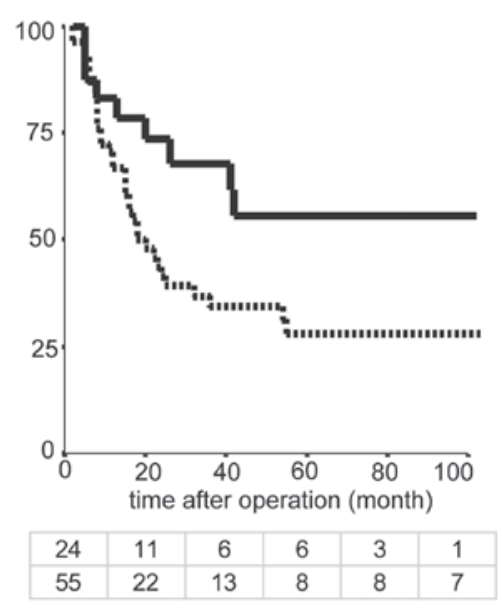

Figure 4. Subgroup analysis based on prognostic factors. Relapse-free survival curves of the MHC class I-negative cases according to adjuvant therapy groups: PSK group (solid line) and chemotherapy-only (control) group (dash line). Survival in patients with (A) diffuse-type carcinoma, (B) venous invasion and (C) serosal infiltration demonstrated improved 3-year prognosis in the PSK group compared to the control group. (D) In patients with $\geq 3$ lymph node metastases, the PSK group demonstrated a significantly improved prognosis than the control group. PSK, protein-bound polysaccharide K.

prognosis have been reported to be correlated in various types of cancer, including breast, lung, renal cell carcinoma and colon cancer $(8,9,17-19)$. The present study found that the expression rate of MHC class I in primary gastric cancer lesions was $55 \%$, close to the values that have been reported in other studies (20). In addition, we identified that decreased MHC class I expression correlated with diffuse-type tumor histology, supporting previous findings (7). Although the correlation between diffuse-type gastric cancer and $\mathrm{MHC}$ class I expression is unclear, immune responses of CTLs due to Fas-Fas-L were reported to be decreased in diffuse-type gastric cancer (21).

Various studies have examined the correlation between MHC class I expression in the primary lesion and lymph node metastasis. In esophageal cancer, MHC class I expression in metastatic lymph nodes was found to be depressed compared to that in the primary lesion (9). That is, cancer cells that have evaded the host immune response are considered to be able to readily form metastatic foci in the lymph nodes.

Expression of MHC class I on the surface of cancer cells decreases as a result of loss of heterozygosity and paucity of $\beta 2$-microglobulin. Conversely, recovery of MHC class I expression was reported as a result of interferon- $\gamma$ production by $\mathrm{T}$ cells and natural killer cells (22). Therefore, loss of MHC class I expression can be considered reversible.

PSK induces production of interleukin 8 by reacting with circulating monocytes, and also activates CTLs and causes dendritic cells to mature $(3,23,24)$. In addition, PSK may be able to enhance MHC class I expression by tumor cells (23). Immunocompetent cells that have been activated by PSK act on trace residual gastric cancer cells of which MHC class I expression has been reduced and bring about recovery of that expression. The increased expression of MHC class I on the surface of cancer cells makes it easier for CTLs to attack the cancer cells and possibly eliminate them. A clinical study of PSK in combination with adjuvant chemotherapy for colorectal cancer found that prognosis was improved for patients with lymph node metastases and patients with a high degree of lymphatic invasion (25). We therefore consider that the survival-prolonging effects observed in the gastric cancer patients with lymph node metastasis in this study were attributable to PSK. 
In conclusion, the present findings indicate that survival-prolonging effects can be expected when PSK is included in the therapy for gastric cancer patients who have undergone curative resection and are at high risk of recurrence due to the presence of lymph node metastasis and the absence of MHC class I expression. PSK can be surmised to exert effects on residual cancer cells that have evaded the antitumor immune functions, and also has the potential to prevent lymph node metastasis. However, PSK cannot be expected to be effective in all gastric cancer patients, and identification of biomarkers to identify potential responders is required.

\section{References}

1. Sakuramoto S, Sasako M, Yamaguchi T, et al: Adjuvant chemotherapy for gastric cancer with S-1, an oral fluoropyrimidine. N Engl J Med 357: 1810-1820, 2007.

2. Zhang H, Morisaki T, Matsunaga H, et al: Protein-bound polysaccharide PSK inhibits tumor invasiveness by down-regulation of TGF-beta1 and MMPs. Clin Exp Metastasis 18: 343-352, 2000.

3. Kanazawa M, Yoshihara K, Abe H, et al: Effects of PSK on T and dendritic cell differentiation in gastric or colorectal cancer patients. Anticancer Res 25: 443-449, 2005.

4. Zhang H, Morisaki T, Nakahara C, et al: PSK-mediated NF-kappaB inhibition augments docetaxel-induced apoptosis in human pancreatic cancer cells NOR-P1. Oncogene 22: 2088-2096, 2003.

5. Nakazato H, Koike A, Saji S, Ogawa N and Sakamoto J: Efficacy of immunochemotherapy as adjuvant treatment after curative resection of gastric cancer. Study group of immunochemotherapy with PSK for gastric cancer. Lancet 343: 1122-1126, 1994.

6. Cabrera T, Lara E, Romero JM, et al: HLA class I expression in metastatic melanoma correlates with tumor development during autologous vaccination. Cancer Immunol Immunother 56: 709-717, 2007.

7. Ishigami S, Natsugoe S, Nakajo A, Arigami T, Kitazono M, Okumura H, Matsumoto M, Uchikado Y, Setoyama T, Sasaki K and Aikou T: HLA-class I expression in gastric cancer. J Surg Oncol 97: 605-608, 2008.

8. Lee HW, Min SK, Ju YS, et al: Prognostic significance of HLA class I expressing in gastric carcinoma defined by monoclonal anti-pan HLA class I antibody, EMR8-5. J Gastrointest Surg 15: 1336-1343, 2011

9. Liu Q, Hao C, Su P and Shi J: Down-regulation of HLA class I antigen-processing machinery components in esophageal squamous cell carcinomas: association with disease progression. Scand J Gastroenterol 44: 960-969, 2009.

10. Shimada H, Okazumi S, Koyama M and Murakami K: Japanese Gastric Cancer Association Task Force for Research Promotion: Clinical utility of ${ }^{18} \mathrm{~F}$-fluoro-2-deoxyglucose positron emission tomography in gastric cancer. A systematic review of the literature. Gastric Cancer 14: 13-21, 2011.
11. Roder JD, Bottcher K, Siewert JR, Busch R, Hermanek P and Meyer HJ: Prognostic factors in gastric carcinoma. Results of the German gastric carcinoma study 1992. Cancer 72: 2089-2097, 1993.

12. Sakcak I, Yildiz BD, Avsar FM, et al: Does N ratio affect survival in D1 and D2 lymph node dissection for gastric cancer? World J Gastroenterol 17: 4007-4012, 2011.

13. Kim JS, Kim MA, Oh DY, et al: Increasing nodal ratio is a poor prognostic factor for survival in stage III-IV (M0) gastric cancer patients who received curative surgery followed by adjuvant chemotherapy: a retrospective study. Jpn J Clin Oncol 41: 245-252, 2011.

14. Saito H, Fukumoto Y, Osaki T, et al: Prognostic significance of level and number of lymph node metastases in patients with gastric cancer. J Surg Oncol 14: 1688-1693, 2007.

15. Funada T, Kochi M, Yamazaki S, Fujii M and Takayama T: Prognostic significance of a new system for categorization of the number of lymph node metastases in gastric cancer. Hepatogastroenterology 58: 642-646, 2011.

16. Rosenberg SA: Progress in human tumour immunology and immunotherapy. Nature 411: 380-384, 2001.

17. Mizukami Y, Kono K, Maruyama T, et al: Downregulation of HLA class I molecules in the tumour is associated with a poor prognosis in patients with oesophageal squamous cell carcinoma. Br J Cancer 99: 1462-1467, 2008.

18. Seliger B, Hohne A, Knuth A, et al: Analysis of the major histocompatibility complex class I antigen presentation machinery in normal and malignant renal cells: evidence for deficiencies associated with transformation and progression. Cancer Res 56: 1756-1760, 1996

19. Simpson JA, Al-Attar A, Watson NF, Scholefield JH, Ilyas M and Durrant LG: Intratumoral T cell infiltration, MHC class I and STAT1 as biomarkers of good prognosis in colorectal cancer. Gut 59: 926-933, 2010.

20. Shen YQ, Zhang JQ, Miao FQ, et al: Relationship between the downregulation of HLA class I antigen and clinicopathological significance in gastric cancer. World J Gastroenterol 11: 3628-3631, 2005.

21. Lee TB, Min YD, Lim SC, et al: Fas (Apo-1/CD95) and Fas ligand interaction between gastric cancer cells and immune cells. J Gastroenterol Hepatol 17: 32-38, 2002.

22. Zhou F: Molecular mechanisms of IFN-gamma to up-regulate MHC class I antigen processing and presentation. Int Rev Immunol 28: 239-260, 2009.

23. Vanky F, Wang P and Klein E: The polysaccharide K (PSK) potentiates in vitro activation of the cytotoxic function in human blood lymphocytes by autologous tumour cells. Cancer Immunol Immunother 35: 193-198, 1992.

24. Pedrinaci S, Algarra I and Garrido F: Protein-bound polysaccharide (PSK) induces cytotoxic activity in the NKL human natural killer cell line. Int J Clin Lab Res 29: 135-140, 1999.

25. Sakai T, Yamashita Y, Maekawa T, Mikami K, Hoshino S and Shirakusa T: Immunochemotherapy with PSK and fluoropyrimidines improves long-term prognosis for curatively resected colorectal cancer. Cancer Biother Radiopharm 23: 461-467, 2008. 